\title{
Review Article \\ Elastic Stability of Annular Thin Plates with One Free Edge
}

\author{
Nagarjuna Jillella and John Peddieson \\ Department of Mechanical Engineering, Tennessee Technological University, 115 West 10th Street, Box 5014, Cookeville, TN 38505 , USA
}

Correspondence should be addressed to John Peddieson; jpeddieson@tntech.edu

Received 21 April 2013; Accepted 20 June 2013

Academic Editor: Lucio Nobile

Copyright (C) 2013 N. Jillella and J. Peddieson. This is an open access article distributed under the Creative Commons Attribution License, which permits unrestricted use, distribution, and reproduction in any medium, provided the original work is properly cited.

\begin{abstract}
The elastic stability of annular thin plates having one free edge and subjected to axisymmetric radial edge loads at the other edge is investigated. The supported edge is allowed to be either simply supported or clamped against axial (transverse) deflection. Both compression buckling and tension buckling (wrinkling) are investigated. To insure accuracy, two methods of solving the appropriate eigenvalue problems are used and found to yield essentially identical results. A selection of these results for both compression and tension buckling is presented graphically and used to illustrate interesting aspects of the solutions.
\end{abstract}

\section{Introduction}

This paper deals with the elastic stability of an annular thin plate subjected to axisymmetric in-plane edge loads. The case of equal compressive loads applied at both boundaries was dealt with definitively by Yamaki [1]. In this case the in-plane radial and circumferential stress resultants are uniform and equal, producing a closed-form transcendental equation from which the buckling loads can be deduced. If the in-plane boundary loads (either tensile or compressive) are unequal the in-plane stress resultants are variable and unequal, creating a more complicated situation in which numerical work is normally required. Various aspects of this general problem have been investigated by Timoshenko and Gere [2], Mansfield [3], Majumdar [4], Yu and Zhang [5], Machinek and Troger [6], Coman and Haughton [7, 8], Coman and Bassom $[9,10]$, Noh et al. [11], and Jillella and Peddieson [12]. A particularly interesting facet of this class of problems is that radial tensile loads can produce compressive circumferential stress resultants over a portion of the plate that lead to radial wrinkling (tension buckling). It should be noted that wrinkling analysis has often been carried out using tension field theory (see, for instance, Coman [13] for a recent discussion). Since that approach is not used in this work, the vast pertinent literature will not be reviewed herein.

The present paper reports an investigation of the elastic stability of an annular thin plate having one load-free edge.
The other edge is subjected to uniform radial tension or compression and is either simply supported or clamped against axial deflection. This investigation both overlaps some findings of the papers referenced above (as discussed subsequently) and adds significant new information.

The remainder of the paper is organized as follows. First, the pertinent governing equations are reviewed. Next, a selection of results for both compression and tension buckling is presented and discussed. Finally, a summary of the work and a recapitulation of important conclusions are given.

\section{Governing Equations}

In this section the governing equations are reviewed for the bending of thin, elastic, homogeneous, isotropic annular plates of uniform thickness undergoing small deflections and subjected to axisymmetric in-plane loads (see, for instance, Timoshenko and Gere [2] for more details). Consider an annular plate having respective inner and outer radii $r_{i}$ and $r_{o}$ and thickness $h$. The plate is assumed to be linearly elastic with Young's modulus $E$ and Poisson's ratio $v$. It is convenient to describe the problem in terms of the respective radial, azimuthal (circumferential), and axial cylindrical polar coordinates $r, \theta$, and $z$ with the plane $z=0$ being coincident 
with the plate's middle surface and the $z$ axis being the axis of symmetry. The appropriate differential equation is

$$
D \nabla^{4} w-\left(N_{r} \partial_{r r} w+N_{\theta}\left(\frac{\partial_{r} w}{r}+\frac{\partial_{\theta \theta} w}{r^{2}}\right)\right)=0
$$

where $w$ is the axial (transverse) displacement,

$$
\nabla^{2} w=\partial_{r r} w+\frac{\partial_{r} w}{r}+\frac{\partial_{\theta \theta} w}{r^{2}}
$$

is the two dimensional Laplacian operator,

$$
D=\frac{E h^{3}}{12\left(1-v^{2}\right)}
$$

is the plate modulus, $N_{r}$ is the radial normal force resultant, and $N_{\theta}$ is the circumferential normal force resultant. In all equations appearing in the present work partial derivative operators are understood to operate only on the immediately following symbol. The normal force resultants are found by solving the uncoupled plane stress problem associated with axisymmetric radial loading at the inner and outer boundaries (see, for instance, [10]) to get

$$
\begin{gathered}
N_{r}=\frac{N_{i}\left(\left(r_{o} / r\right)^{2}-1\right)+N_{o}\left(\left(r_{o} / r_{i}\right)^{2}-\left(r_{o} / r\right)^{2}\right)}{\left(r_{o} / r_{i}\right)^{2}-1}, \\
N_{\theta}=\frac{-N_{i}\left(\left(r_{o} / r\right)^{2}+1\right)+N_{o}\left(\left(r_{o} / r_{i}\right)^{2}+\left(r_{o} / r\right)^{2}\right)}{\left(r_{o} / r_{i}\right)^{2}-1},
\end{gathered}
$$

where $N_{i}$ and $N_{o}$ are the respective inner and outer boundary tensile force resultants. Support conditions to be employed subsequently are

$$
\begin{aligned}
& \text { clamped: } w=0, \quad \partial_{r} w=0, \\
& \text { simply supported: } w=0, \quad M_{r}=0, \\
& \text { free: } M_{r}=0, \quad V_{r}=0,
\end{aligned}
$$

where

$$
M_{r}=D\left(\partial_{r r} w+v\left(\frac{\partial_{r} w}{r}+\frac{\partial_{\theta \theta} w}{r^{2}}\right)\right)
$$

is the radial bending moment resultant and

$$
\begin{gathered}
V_{r}=-D\left(\partial_{r r r} w+\frac{\partial_{r r} w}{r}-\frac{\partial_{r} w}{r^{2}}-\frac{(3-v) \partial_{\theta \theta} w}{r^{3}}\right. \\
\left.+\frac{(2-v) \partial_{r \theta \theta} w}{r^{2}}\right)+N_{r} \partial_{r} w
\end{gathered}
$$

is the radial Kirchhoff shear force resultant.

For the purpose of numerical analysis it is convenient to convert the equations to dimensionless forms. Towards this end it is helpful to define dimensionless quantities (denoted by superposed asterisks) through the equations

$$
\begin{aligned}
r=r_{o} r^{*}, & r_{i}=r_{o} r_{i}^{*}, \quad w=h w^{*}, \quad M_{r}=\frac{D h M_{r}^{*}}{r_{o}^{2}}, \\
M_{\theta}=\frac{D h M_{\theta}^{*}}{r_{o}^{2}}, & V_{r}=\frac{D h V_{r}^{*}}{r_{o}^{3}}, \\
N_{r}=N_{c} N_{r}^{*}, & N_{\theta}=N_{c} N_{\theta}^{*}, \\
N_{i}=-N_{c} n_{i}^{*}, & N_{o}=-N_{c} n_{o}^{*},
\end{aligned}
$$

where $N_{c}$ is a characteristic in-plane load. When (8) are substituted into the appropriate equations discussed previously and, for simplicity, the asterisks are dropped from the results (with the understanding that all subsequent equations, except (14), are in terms of dimensionless quantities) the results are

$$
\begin{gathered}
\nabla^{4} w-\lambda^{2}\left(N_{r} \partial_{r r} w+N_{\theta}\left(\frac{\partial_{r} w}{r}+\frac{\partial_{\theta \theta} w}{r^{2}}\right)\right)=0, \\
N_{r}=-\frac{n_{i}\left(1 / r^{2}-1\right)+n_{o}\left(1 / r_{i}^{2}-1 / r^{2}\right)}{1 / r_{i}^{2}-1}, \\
N_{\theta}=\frac{n_{i}\left(1 / r^{2}+1\right)-n_{o}\left(1 / r_{i}^{2}+1 / r^{2}\right)}{1 / r_{i}^{2}-1}, \\
M_{r}=\partial_{r r} w+v\left(\frac{\partial_{r} w}{r}+\frac{\partial_{\theta \theta} w}{r^{2}}\right), \\
V_{r}=-\left(\partial_{r r r} w+\frac{\partial_{r r} w}{r}-\frac{\partial_{r} w}{r^{2}}-\frac{(3-v) \partial_{\theta \theta} w}{r^{3}}\right. \\
\left.+\frac{(2-v) \partial_{r \theta \theta} w}{r^{2}}\right)+\lambda^{2} N_{r} \partial_{r} w,
\end{gathered}
$$

where

$$
\lambda^{2}=\frac{N_{c} r_{o}^{2}}{D}
$$

defines an in-plane load dimensionless parameter, which can be thought of as a dimensionless buckling load.

Substituting

$$
w=\sum_{n=0}^{\infty} w_{n} \cos (n \theta)
$$

into (9) leads to the sequence of one dimensional problems

$$
\begin{aligned}
w_{n}^{\prime \prime \prime \prime}+ & \frac{2 w_{n}^{\prime \prime \prime}}{r}-\left(\frac{2 n^{2}+1}{r^{2}}+\lambda^{2} N_{r}\right) w_{n}^{\prime \prime} \\
+ & \frac{\left(\left(2 n^{2}+1\right) / r^{2}-\lambda^{2} N_{\theta}\right) w_{n}^{\prime}}{r} \\
+\frac{n^{2}\left(\left(n^{2}-4\right) / r^{2}+\lambda^{2} N_{\theta}\right) w_{n}}{r^{2}} & =0
\end{aligned}
$$


with a prime denoting differentiation with respect to $r$. In a similar manner, substituting (15) into (12) and (13) yields the corresponding Fourier cosine series coefficients

$$
\begin{gathered}
M_{r, n}=w_{n}^{\prime \prime}+v\left(\frac{w_{n}^{\prime}}{r}-\frac{n^{2} w_{n}}{r^{2}}\right) \\
V_{r, n}=-\left(w_{n}^{\prime \prime \prime}+\frac{w_{n}^{\prime \prime}}{r}-\left(\frac{1}{r^{2}}+n^{2}(2-v)+\lambda^{2} N_{r}\right) w_{n}^{\prime}\right. \\
\left.+\frac{n^{2}(3-v) w_{n}}{r^{3}}\right)
\end{gathered}
$$

which, when substituted into (5), produce the boundary conditions

$$
\begin{gathered}
\text { clamped: } w_{n}=0, \quad w_{n}^{\prime}=0, \\
\text { simply supported: } w_{n}=0, \quad M_{r, n}=0, \\
\text { free: } M_{r, n}=0, \quad V_{r, n}=0 .
\end{gathered}
$$

Equation (16) and an appropriate combination of (19)(21) constitute an eigenvalue problem for the dimensionless buckling parameter $\lambda$ which must be solved numerically for the configurations to be considered below. To insure accuracy, two independent methods of doing this were employed. The first was a variant of the compound matrix method used by Coman and Haughton [7, 8] and the second was the imperfection method used by Jillella and Peddieson [12]. The interested reader is referred to these publications and the corresponding references cited therein for the details of the two approaches. In all cases the predictions of the two procedures were essentially identical. A representative sample of these predictions is presented in the next two sections.

\section{Results for Load-Free Inner Edge}

Majumdar [4] considered the problem of compression buckling of a free/clamped annular plate having a uniform radial compressive loading at the outer edge and no radial loading at the inner edge (load-free inner edge). This is a generalization of the problem discussed by Timoshenko and Gere [2] who dealt with only axisymmetric buckling. Majumdar [4] found that the cases of $n=0$ (axisymmetric) and $n=1$ (one circumferential node) produced exact closed-form transcendental equations in terms of Bessel functions from which the buckling loads could be determined, with the former yielding results in agreement with those obtained by Meissner quoted in [2]. For $n \geq 2$, no exact closed-form transcendental equations could be found and estimates of the buckling loads were made using the Rayleigh/Ritz method. Machinek and Troger [6], in preparation for a postbuckling study, reported limited results based on numerical solutions of the exact eigenvalue problem for $n \geq 2$. Coman and Bassom [10], in preparation for an analytical study employing asymptotic methods, recently reproduced these numerical results. No other work on the load-free inner edge configuration appears to have been published. For this reason, it was decided to investigate a set of configurations involving an annular plate with a load-free inner edge. These have either uniform tension or compression acting on either a clamped or simply supported outer edge.

In the present notation a radial loading involving a free inner edge and uniform tension or compression at the outer edge corresponds to

$$
n_{i}=0, \quad n_{o}= \pm 1
$$

(with the top sign indicating compression and the bottom sign tension) which, according to (10) and (11), produces

$$
\begin{aligned}
& N_{r}=\mp \frac{1 / r_{i}^{2}-1 / r^{2}}{1 / r_{i}^{2}-1}, \\
& N_{\theta}=\mp \frac{1 / r_{i}^{2}+1 / r^{2}}{1 / r_{i}^{2}-1} .
\end{aligned}
$$

Here the quantity $N_{c}$ stands for the magnitude of the dimensional outer edge stress resultant. It can be seen from (23) that both stress resultants have the same sign for all $r$. Thus radial compression produces circumferential compression and radial tension produces circumferential tension. In this configuration, therefore, tension buckling is impossible and results will be presented for compression buckling only.

Predictions relevant to compression buckling are reported in Figures 1, 2, 3, and 4 for free/clamped support conditions. Figure 1 shows curves of buckling load versus inner radius associated with the first eleven circumferential buckling modes for $v=1 / 3$, the value thought to be used by Majumdar [4]. A value of $v$ is not explicitly stated in [4], but $v=1 / 3$ is used to obtain the results reported in [2] to which comparisons are made in [4]. The composite curve created by combining the lowest portions of all individual curves contained in Figure 1 (and subsequent similar figures) indicates the lowest buckling load for any given value of the dimensionless inner radius (radius ratio). In addition, for plates exhibiting initial imperfections, each individual curve characterizes the buckling behavior associated with a particular circumferential imperfection pattern (see, for instance, [2] for a detailed discussion). Thus, for example, it is expected that the buckling loads associated with an axisymmetric imperfection pattern would be determined from the curve labeled $n=0$ in Figure 1 even though they are not always the lowest possible buckling loads.

The curves corresponding to $n=0$ and 1 depicted in Figure 1 can be directly compared with Figure 1 of [4] and agree well with the accuracy of visual inspection. It is interesting to note that for $n=0$, the trend of a small decrease in the value of the buckling load for the smaller inner radii is captured by the numerical method. Rayleigh/Ritz predictions of buckling loads for $0 \leq n \leq 6$ and $n=10$ are presented in Figure 2 of [4]. The curve for $n=0$ fails to capture the decrease in $\lambda^{2}$ discussed above. In addition, the energy method predicts the lowest buckling load at $r_{i}=0.5$ to correspond to $n=1$ rather than to $n=0$ as indicated by the exact solution. This effect is also captured by the current methodology, as illustrated by Figure 1. 


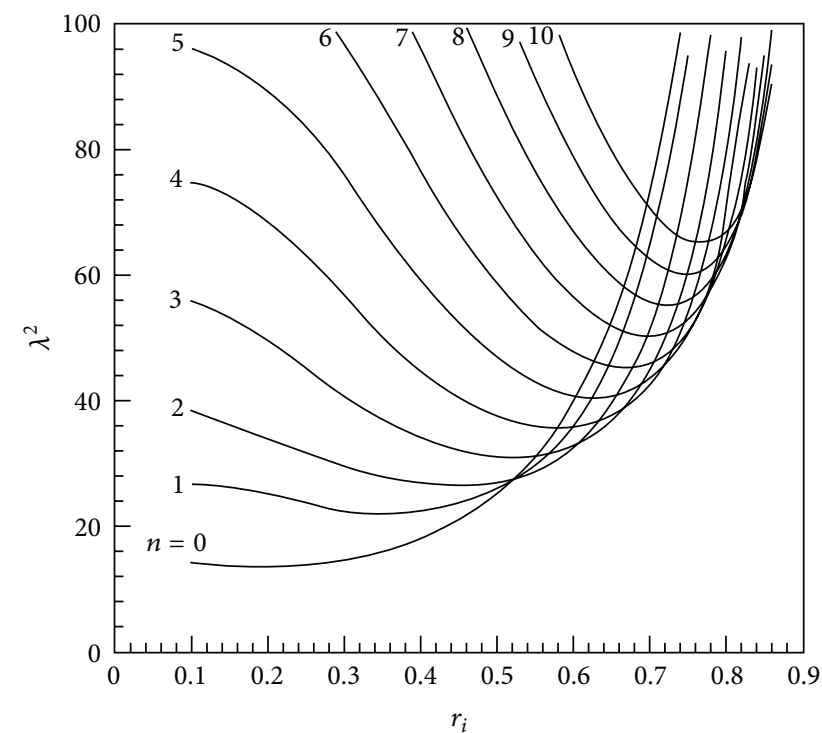

FIGURE 1: Buckling load versus inner radius (free/clamped, $n_{i}=0$, $\left.n_{o}=1, v=1 / 3\right)$.

As stated earlier, the composite curve formed by combining the lowest portions of curves for each circumferential buckling mode (value of $n$ ) indicates the lowest buckling load. It is, therefore, possible that the composite curve associated with the Rayleigh/Ritz method could be an accurate representation of the buckling behavior even if some individual components of the composite curve are inaccurate. This proposition was tested by comparing the lowest buckling loads for selected inner radii predicted by both the present numerical method and Figure 2 of [4] (to the accuracy of visual inspection). It was found that the maximum errors associated with the Rayleigh/Ritz method are in the 10\%-15\% range. Thus, the Rayleigh/Ritz composite curve is reasonably accurate.

It is often asserted in the literature that compression buckling loads are insensitive to the value of Poisson's ratio. It is of interest to use the results presented herein to test this assertion. Figures 2, 3, and 4 (and other similar sets of predictions to be presented subsequently) depict the composite curves of minimum buckling load versus inner radius for $v=0.1,0.3$, and 0.5 . The difference between the highest and lowest buckling load values for these three Poisson's ratios at a given inner radius can be expressed as a percentage $P$ of the lowest value. For this set of predictions $P$ was found to be in the $8 \%-37 \%$ range.

Figure 3 can be directly compared with both Figure 4 of [6] and Figure 2(a) of [10]. To the accuracy of visual inspection, agreement between the former and the latter two appears to be excellent, thus providing additional validation of the two independent numerical procedures used herein. Figures 1, 2, 3, and 4 all exhibit a rapid increase in the number of circumferential nodes exhibited by the buckling mode as the inner radius increases. This behavior (here associated with compression buckling) will also be observed in one of the tension buckling configurations to be discussed below.

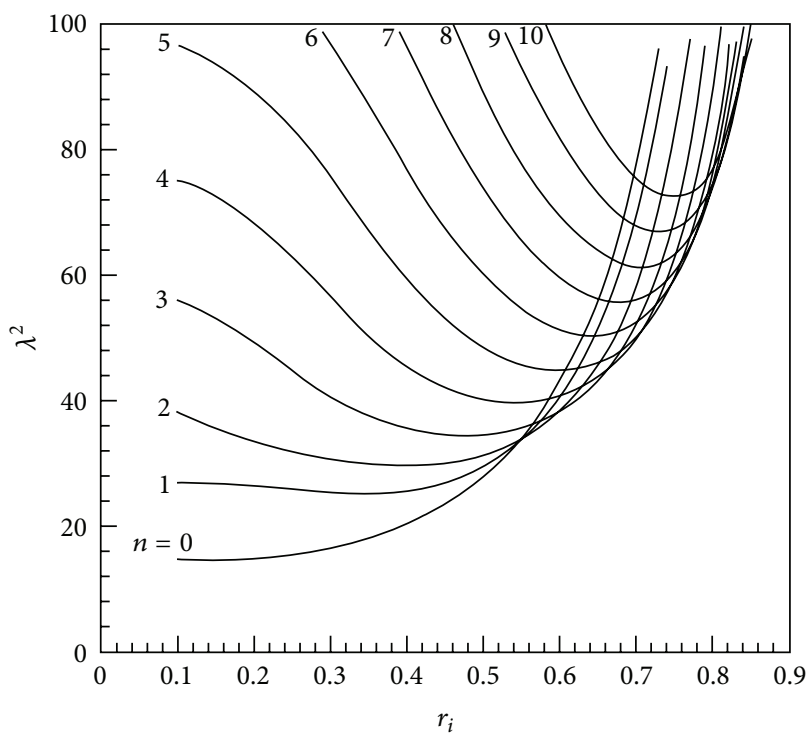

FIgURE 2: Buckling load versus inner radius (free/clamped, $n_{i}=0$, $n_{o}=1, v=0.1$ ).

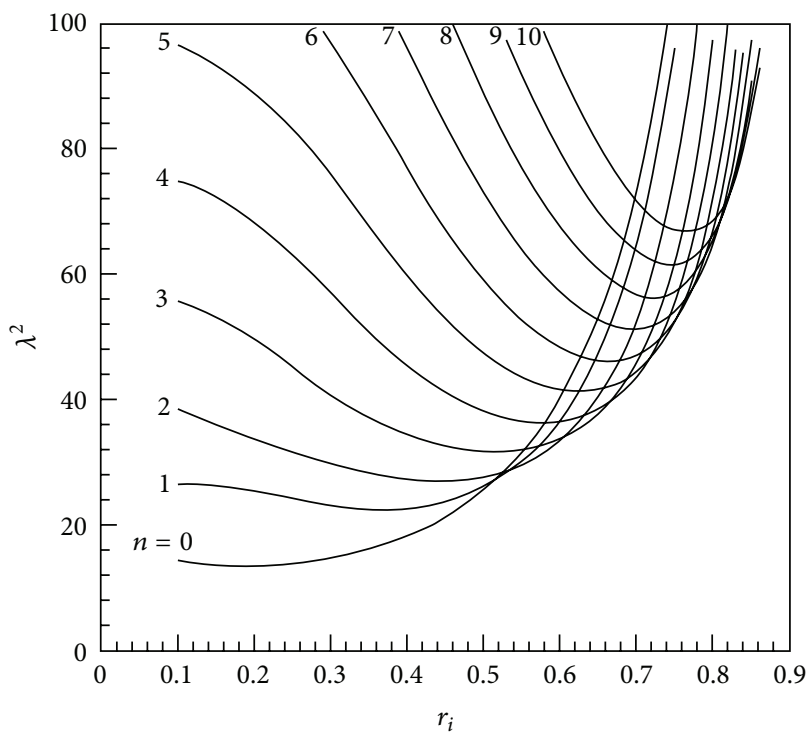

FIGURE 3: Buckling load versus inner radius (free/clamped, $n_{i}=0$, $\left.n_{o}=1, v=0.3\right)$.

Results for compression buckling of the free/simply supported configuration are reported in Figures 5, 6, and 7. It is clear that the lowest buckling load corresponds to axisymmetric buckling for all values of Poisson's ratio. The difference produced by the change from a clamped to simply supported edge is striking. Here $P$ is in the $2 \%-30 \%$ range.

To be consistent with the results presented in [4], predictions were reported above only for cases satisfying the inequality $\lambda^{2} \leq 100$. This fact accounts for the difference between the number of circumferential modes for which information was presented in Figures 1-4 and the number for which it was presented in Figures 5-7. 


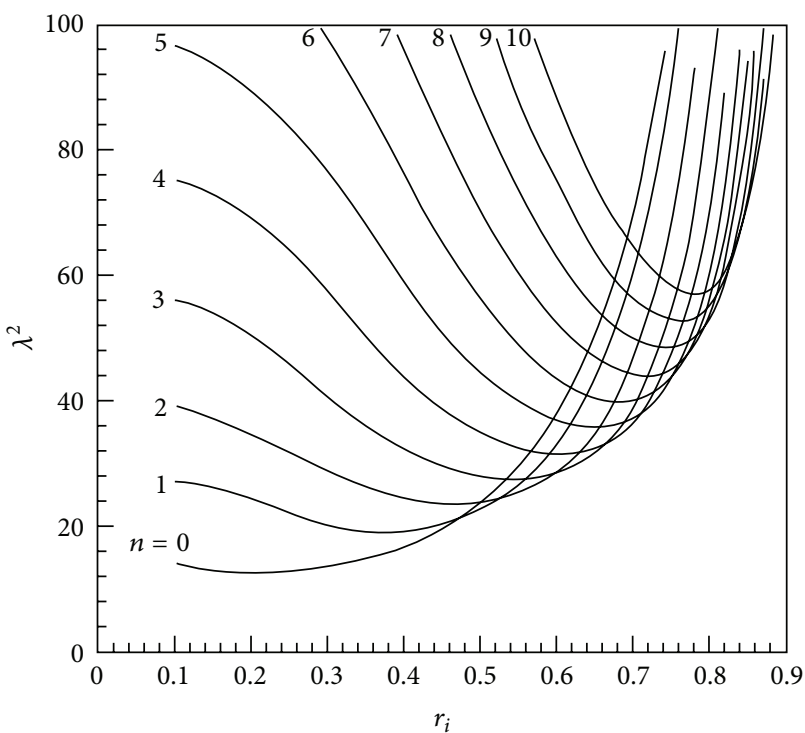

FIGURE 4: Buckling load versus inner radius (free/clamped, $n_{i}=0$, $\left.n_{o}=1, v=0.5\right)$.

\section{Results for Load-Free Outer Edge}

Yu and Zhang [5] considered the problem of tension buckling of a simply supported/free annular plate having a uniform radial tensile loading at the inner edge and no radial loading at the outer edge (load-free outer edge). Yu and Zhang [5] estimated the buckling loads by using the Galerkin method. Coman and Haughton [8], in preparation for an analytical study, presented limited results based on numerical solutions of the exact eigenvalue problem for this configuration. Noh et al. [11] reproduced some of these results using the finite element method. No other work on the load-free outer edge configuration appears to have been published. It was, therefore, decided to investigate a set of configurations involving an annular plate with a load-free outer edge. These have either uniform radial tension or compression acting on either a clamped or simply supported inner edge.

In the present notation a radial loading involving a free outer edge and uniform tension or compression at the inner edge corresponds to

$$
n_{i}= \pm 1, \quad n_{o}=0
$$

(with the top sign indicating compression and the bottom sign tension) which, according to (10) and (11), produces

$$
\begin{aligned}
& N_{r}=\mp \frac{1 / r^{2}-1}{1 / r_{i}^{2}-1}, \\
& N_{\theta}= \pm \frac{1 / r^{2}+1}{1 / r_{i}^{2}-1} .
\end{aligned}
$$

Here $N_{c}$ stands for the magnitude of the dimensional inner edge stress resultant. It can be seen from (25) that the stress resultants have opposite signs for all $r$. Thus radial tension produces circumferential compression and radial

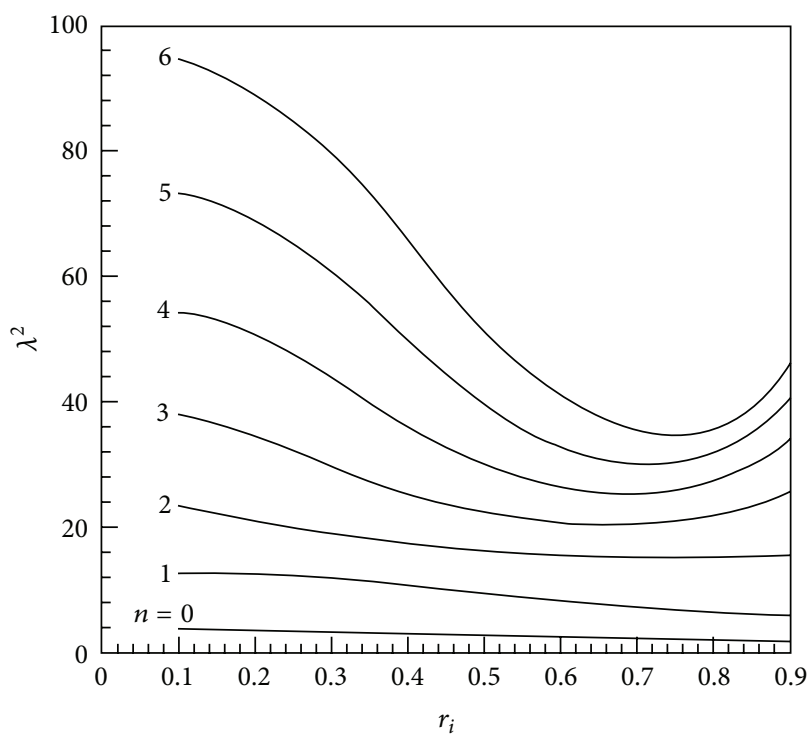

FIGURE 5: Buckling load versus inner radius (free/simply supported, $n_{i}=0, n_{o}=1, v=0.1$ ).

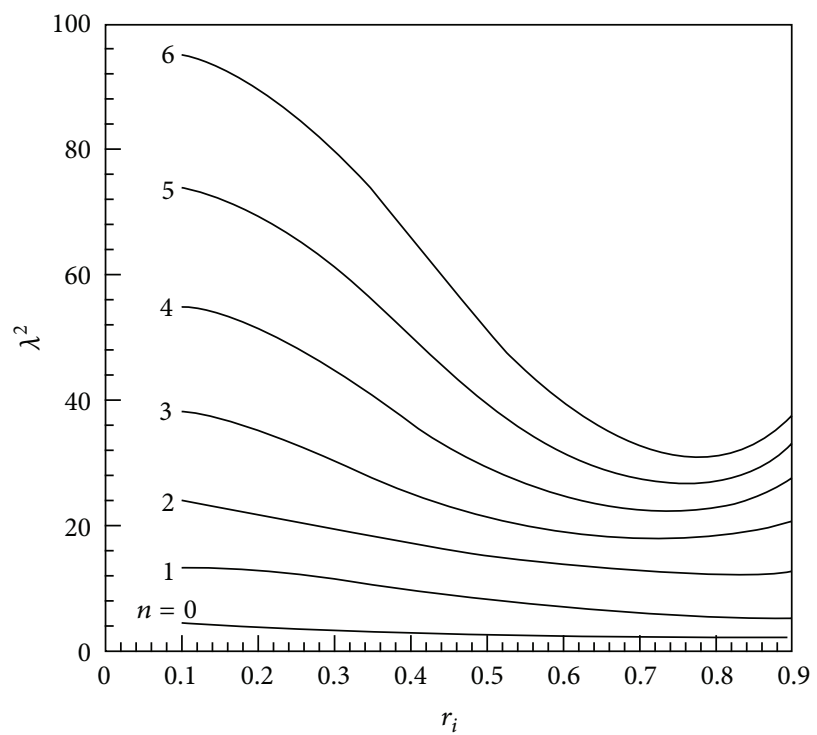

FIGURE 6: Buckling load versus inner radius (free/simply supported, $n_{i}=0, n_{o}=1, v=0.3$ ).

compression produces circumferential tension. In this configuration, therefore, both tension and compression buckling are possible and results for both will be presented.

Figures 8, 9, and 10 show curves of buckling load versus inner radius for several circumferential tension buckling modes associated with the simply supported/free configuration. In this set (and subsequent similar sets) of figures results will be presented only for circumferential buckling modes exhibiting buckling loads in the range $\lambda^{2} \leq 200$ to be consistent with [5]. As before, this will produce different maximum and minimum values of $n$ in different figures. In particular, no buckling was observed in this range for $n=0$ 


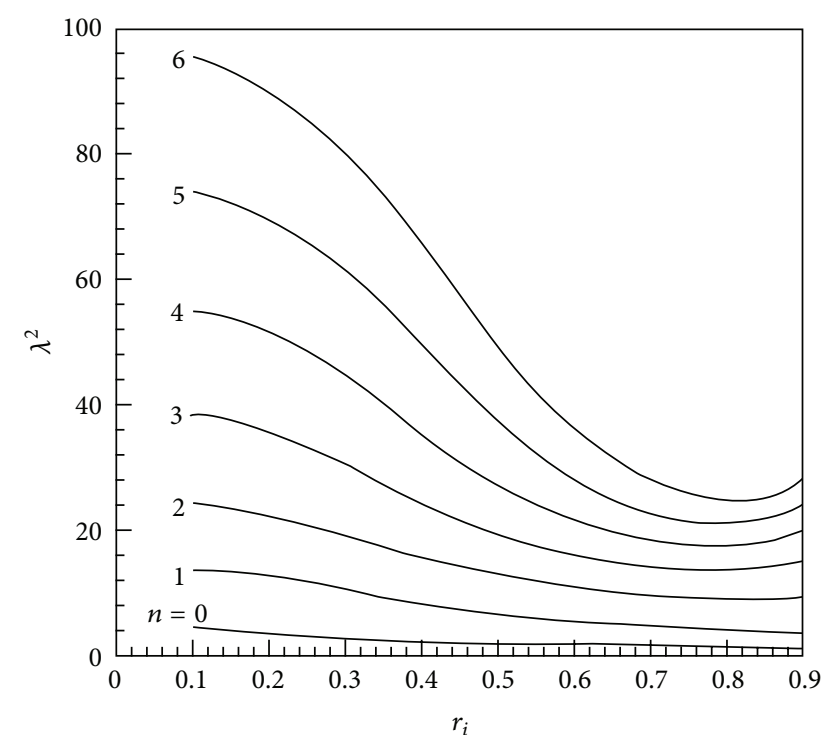

FIGURE 7: Buckling load versus inner radius (free/simply supported, $\left.n_{i}=0, n_{o}=1, v=0.5\right)$.

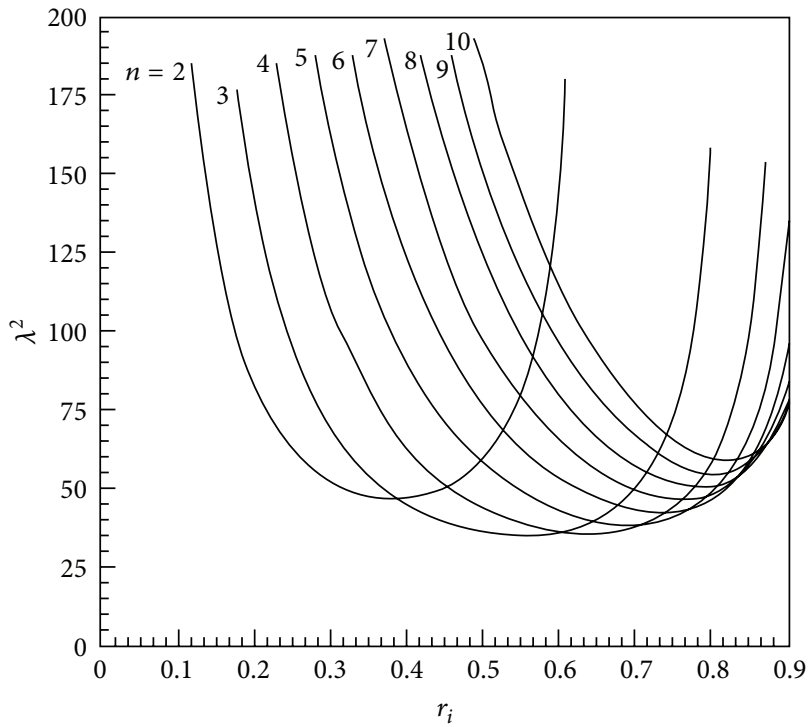

FIGURE 8: Buckling load versus inner radius (simply supported/free, $\left.n_{i}=-1, n_{o}=0, v=0.1\right)$.

or $n=1$, thus accounting for the absence of the associated curves from Figures 8-10.

Figure 9 can be directly compared with the Galerkin predictions presented in Figure 3(a) of [5] for $2 \leq n \leq$ 6. Quantitative comparisons of the results with those read from Figure 3(a) of [5] (to the accuracy of visual inspection) reveal that maximum errors associated with the Galerkin method are in the 20\%-25\% range. Figure 9 can also be compared directly with Figures 3 and 4 of [8]. To the accuracy of visual inspection, agreement seems excellent, providing further validation of the present numerical approaches.

Figures 8-10 illustrate the influence of Poisson's ratio on buckling loads, with $P$ being in the 58\%-67\% range.

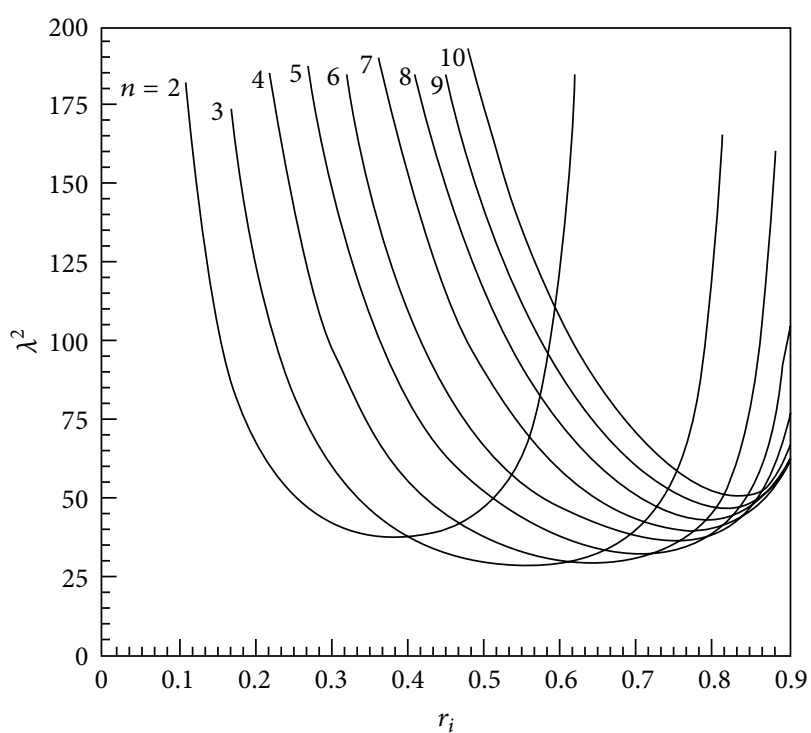

FIGURE 9: Buckling load versus inner radius (simply supported/free, $\left.n_{i}=-1, n_{o}=0, v=0.3\right)$.

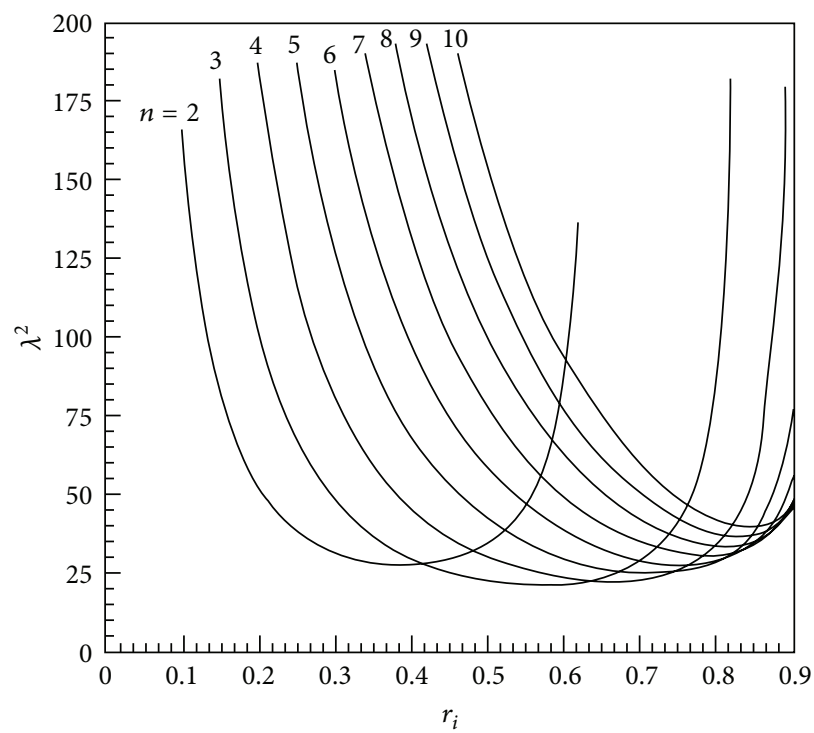

FIGURE 10: Buckling load versus inner radius (simply supported/ free, $n_{i}=-1, n_{o}=0, v=0.5$ ).

These figures also exhibit the rapid increase in buckling mode nodes with increasing inner radius mentioned earlier. Here this phenomenon is associated with tension buckling while previously it was associated with compression buckling. A program of analytical work begun by Coman and Haughton [8] (using Rayleigh's quotient) and continued by Coman and Bassom [9] (using asymptotic methods) reveals that a value of the inner radius exists for each value of $n$ beyond which tension buckling does not occur.

Results for tension buckling of the clamped/free configuration are reported in Figures 11, 12, and 13. While the qualitative influence of Poisson's ratio is slight, $P$ is in the $37 \%-42 \%$ range. Comparison of Figures $8-10$ with 


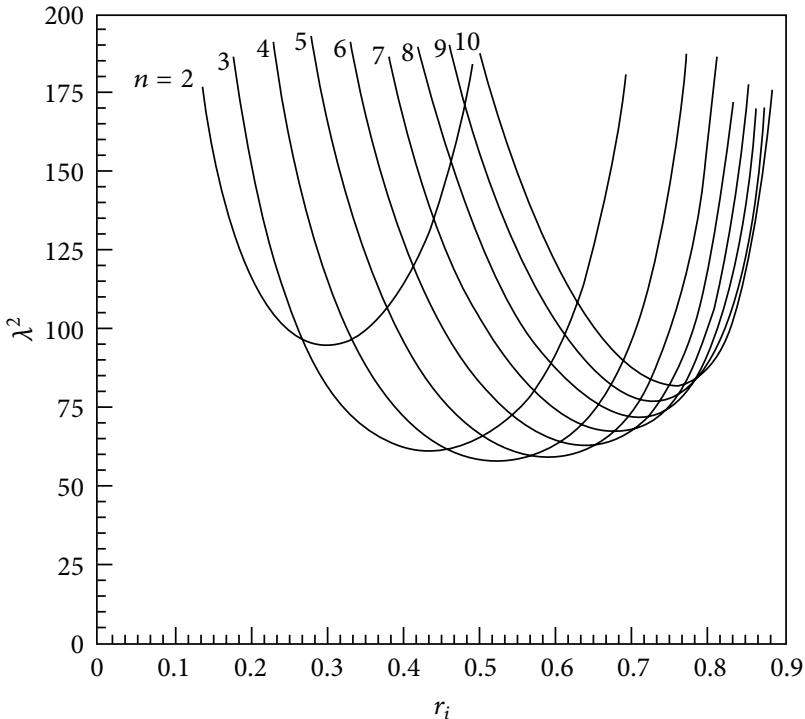

FIGURE 11: Buckling load versus inner radius (clamped/free, $n_{i}=-1$, $\left.n_{o}=0, v=0.1\right)$.

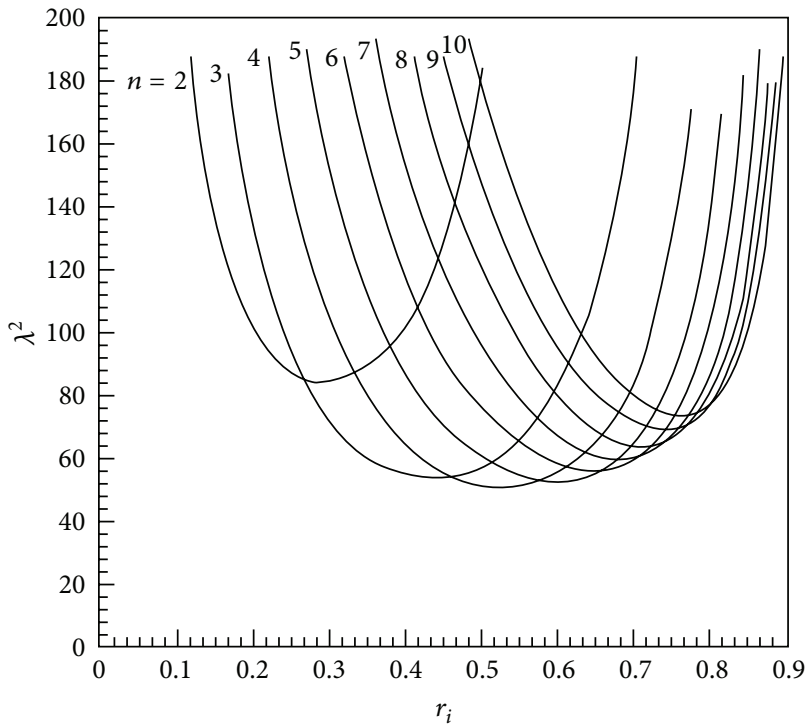

FIGURE 12: Buckling load versus inner radius (clamped/free, $n_{i}=-1$, $\left.n_{o}=0, v=0.3\right)$.

Figures 11-13 suggests that the change from a simply supported to a clamped inner edge does not produce a significant change in the nature of the tension buckling behavior (in sharp contrast to the compression buckling cases discussed earlier). In particular, the rapid increase in buckling mode nodes with increasing inner radius appears in both. It would be interesting to apply the asymptotic analysis of [9] to determine whether there are limiting inner radii above which tension buckling will not occur in this configuration as well. It would also be interesting to investigate the possibility of a unified analytical approach to the rapid node increase phenomenon which appears for both tension and compression buckling.

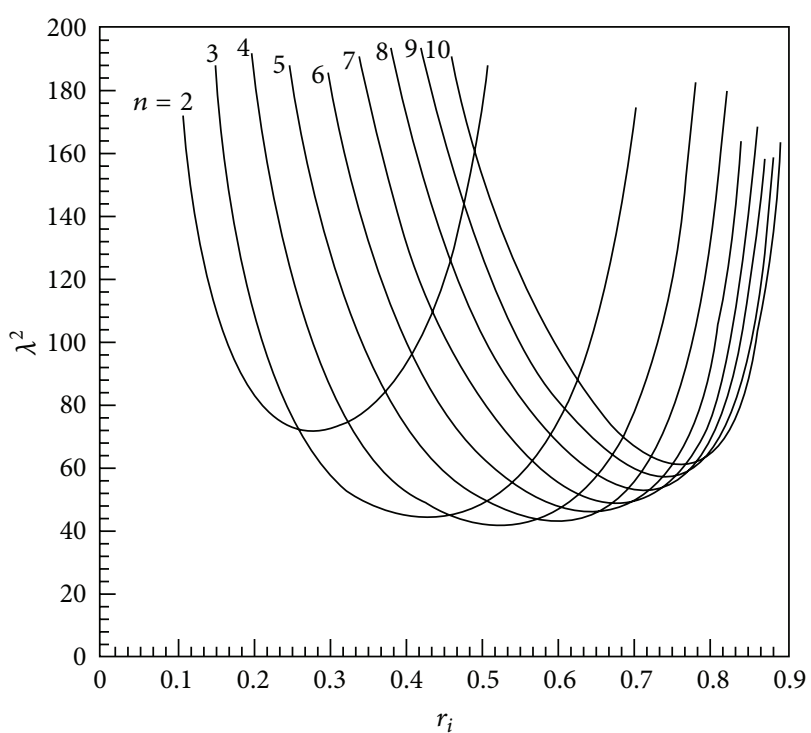

FIGURE 13: Buckling load versus inner radius (clamped/free, $n_{i}=-1$, $n_{o}=0, v=0.5$ ).

As discussed in detail by Jillella and Peddieson [12], the use of a tension field model for wrinkling (tension buckling) analysis does not allow either the dependence on axial support conditions or the wrinkling pattern to be predicted. Examples were given in [12] in which the axial support dependence was significant. Figures 8-13 show that the change from a simply supported to a clamped inner edge does not change the qualitative nature of the wrinkling behavior for a free outer edge but does (as expected) significantly raise the buckling load values. Figures 8-13 also associate a unique value of $n$ with each inner radius for which buckling occurs (except in cases in which two curves cross). This, in turn, quantitatively defines the wrinkle pattern.

Results for compression buckling of the simply supported/free configuration are reported in Figure 14. Since the lowest buckling load corresponds to axisymmetric buckling for all values of Poisson's ratio, one value has been selected as representative for graphical presentation. Additional computed results (not shown) reveal that $P$ is in the $34 \%-44 \%$ range.

Results for compression buckling of the clamped/free configuration are reported in Figure 15. Again, the lowest buckling load corresponds to axisymmetric buckling for all values of Poisson's ratio and one representative value has been chosen for graphical depiction. The results of additional simulations (not shown) indicate that $P$ is in the $8 \%-14 \%$ range. In contrast to the case of tension buckling, the change from a simply supported to a clamped inner edge produces a significant change in the buckling load versus inner radius behavior.

\section{Conclusion}

The foregoing discussed the elastic stability of thin, elastic, homogeneous, isotropic annular plates of uniform thickness. 


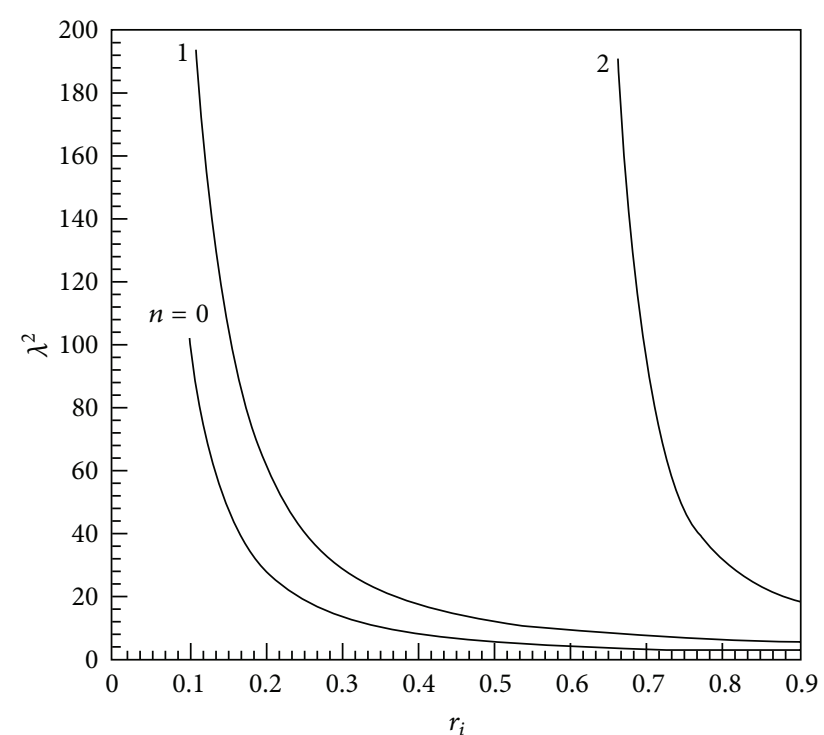

FIGURE 14: Buckling load versus inner radius (simply supported/ free, $n_{i}=1, n_{o}=0, v=0.3$ ).

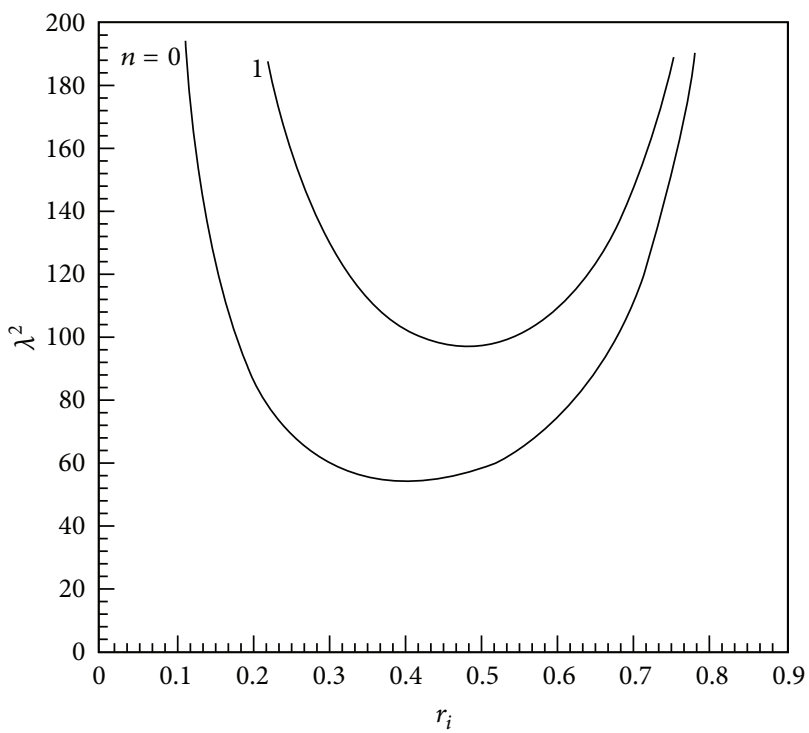

FIGURE 15: Buckling load versus inner radius (clamped/free, $n_{i}=1$, $n_{o}=0, v=0.1$ ).

Two independent numerical approaches were used to solve the eigenvalue problem associated with buckling analysis. Predictions were obtained for several configurations involving a load-free inner or outer edge with axisymmetric radial loading at the opposite edge exhibiting either compression or tension buckling. Two of these were used to verify the numerical approach by comparison with previous published results and several involved new results. Some important conclusions are as follows.

First, some of the results reported herein exhibit significant sensitivities of thin plate buckling loads to Poisson's ratio. Of the configurations investigated, the largest sensitivity was observed for simply supported/free tension buckling while the smallest was observed for clamped/free compression buckling; however no definite pattern is obvious.

Second, standard elastic stability methodology provides a unified approach to both tension and compression buckling of thin plates. In particular, when applied to tension buckling (wrinkling) this approach can make certain quantitative predictions of which tension field theories are incapable. The most important of these are the wrinkle pattern (buckling mode shape) and the effect of axial support conditions. The present work provides examples of such predictions. The papers by Jillella and Peddieson [12] and Coman [13] contain further discussion of the relative merits of tension field and thin plate models for the analysis of flat sheet wrinkling.

Third, the plate thickness enters the dimensionless equations employed herein only through the dimensionless buckling load $\lambda^{2}$ (which depends on the plate modulus $D$ which, in turn, depends on the thickness $h$ ). Thus, no difficulties arise for small thicknesses when using the approaches employed in the present work. Such difficulties are often cited as reasons for preferring tension field models to plate or shell models for the analysis of thin sheet wrinkling. Many commercial finite element codes are based on shear deformation plate and shell models which, of course, sometimes exhibit difficulties in dealing with small thicknesses. These difficulties, however, are generic and not specific to the prediction of wrinkling phenomena.

\section{References}

[1] N. Yamaki, "Buckling of a thin annular plate under uniform compression," Journal of Applied Mechanics, vol. 25, pp. 267$273,1958$.

[2] S. Timoshenko and J. Gere, Theory of Elastic Stability, McGrawHill, New York, NY, USA, 2nd edition, 1960.

[3] E. H. Mansfield, "On the buckling of an annular plate," Quarterly Journal of Mechanics and Applied Mathematics, vol. 13, no. 1, pp. 16-23, 1960.

[4] S. Majumdar, "Buckling of a thin annular plate under uniform compression," AIAA Journal, vol. 9, no. 9, pp. 1701-1707, 1971.

[5] T. X. Yu and L. C. Zhang, "The elastic wrinkling of an annular plate under uniform tension on its inner edge," International Journal of Mechanical Sciences, vol. 28, no. 11, pp. 729-737, 1986.

[6] A. Machinek and H. Troger, "Post-buckling of elastic annular plates at multiple eigenvalues," Dynamics and Stability of Systems, vol. 3, pp. 79-98, 1988.

[7] C. D. Coman and D. M. Haughton, "Localized wrinkling instabilities in radially stretched annular thin films," Acta Mechanica, vol. 185, no. 3-4, pp. 179-200, 2006.

[8] C. D. Coman and D. M. Haughton, "On some approximate methods for the tensile instabilities of thin annular plates," Journal of Engineering Mathematics, vol. 56, no. 1, pp. 79-99, 2006.

[9] C. D. Coman and A. P. Bassom, "Singular behaviour in a generalized boundary eigenvalue problem for annular plates in tension," Quarterly Journal of Mechanics and Applied Mathematics, vol. 60, no. 3, pp. 319-336, 2007.

[10] C. D. Coman and A. P. Bassom, "On a class of buckling problems in a singularly perturbed domain," Quarterly Journal of Mechanics and Applied Mathematics, vol. 62, no. 1, pp. 89-103, 2009. 
[11] S. Noh, M. Abdalla, and W. Faris, "A study of annular plate buckling problem with tension loaded at inner edge," Analele Universitatii Eftimie Murgu, vol. 17, pp. 97-103, 2010.

[12] N. Jillella and J. Peddieson, "Modeling of wrinkling of thin circular sheets," International Journal of Non-Linear Mechanics, vol. 47, no. 1, pp. 85-91, 2012.

[13] C. D. Coman, "On the applicability of tension field theory to a wrinkling instability problem," Acta Mechanica, vol. 190, no. 1-4, pp. 57-72, 2007. 

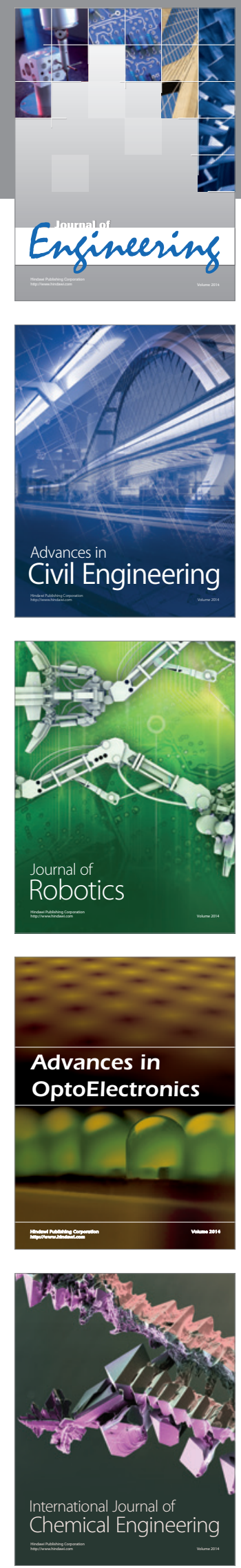

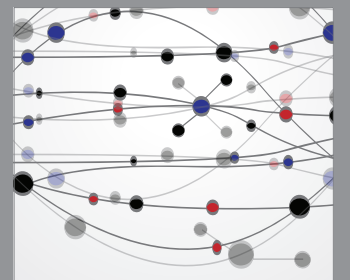

The Scientific World Journal
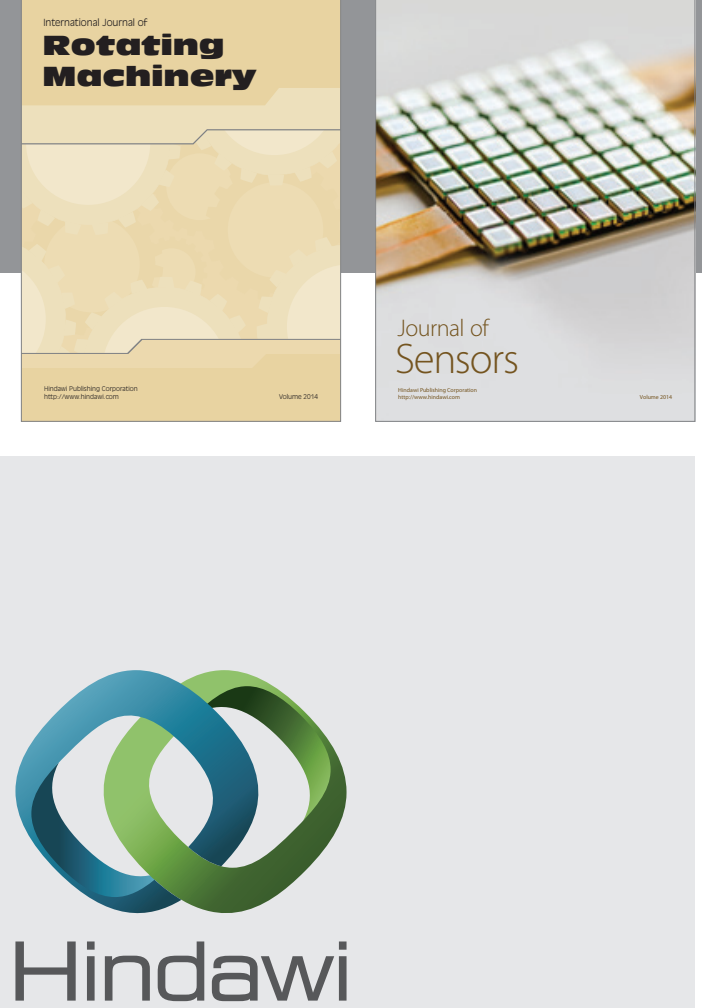

Submit your manuscripts at http://www.hindawi.com
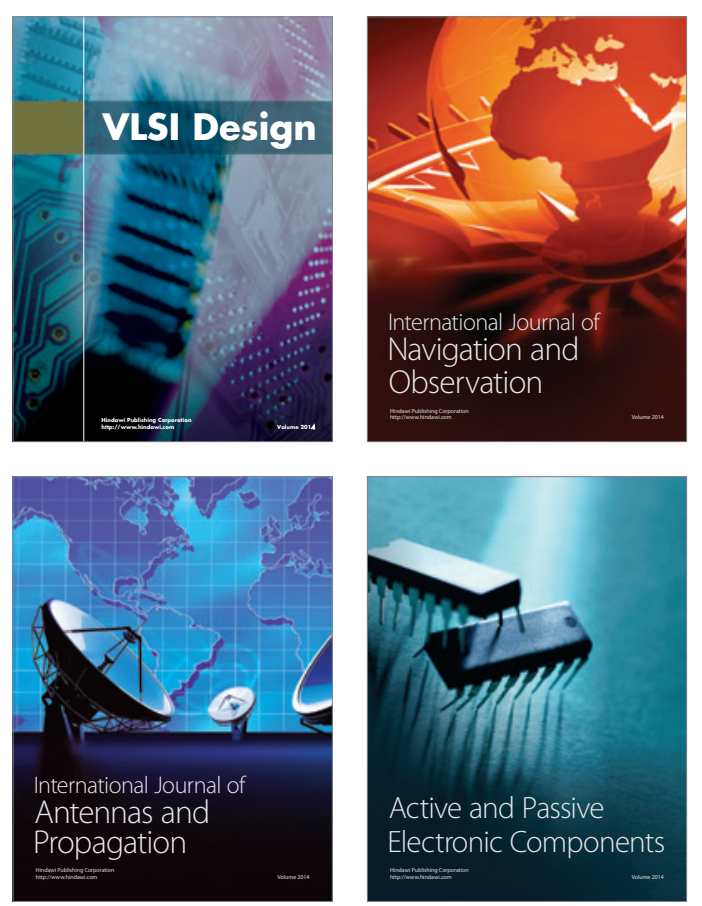
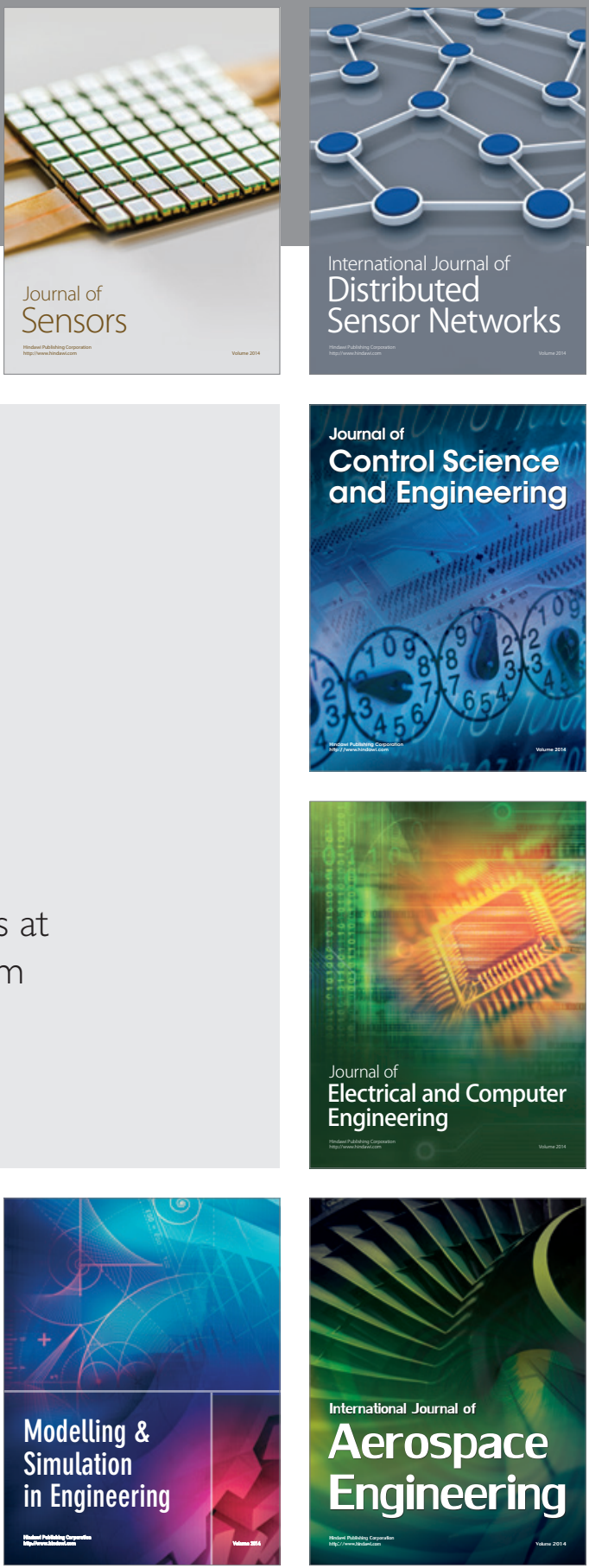

Journal of

Control Science

and Engineering
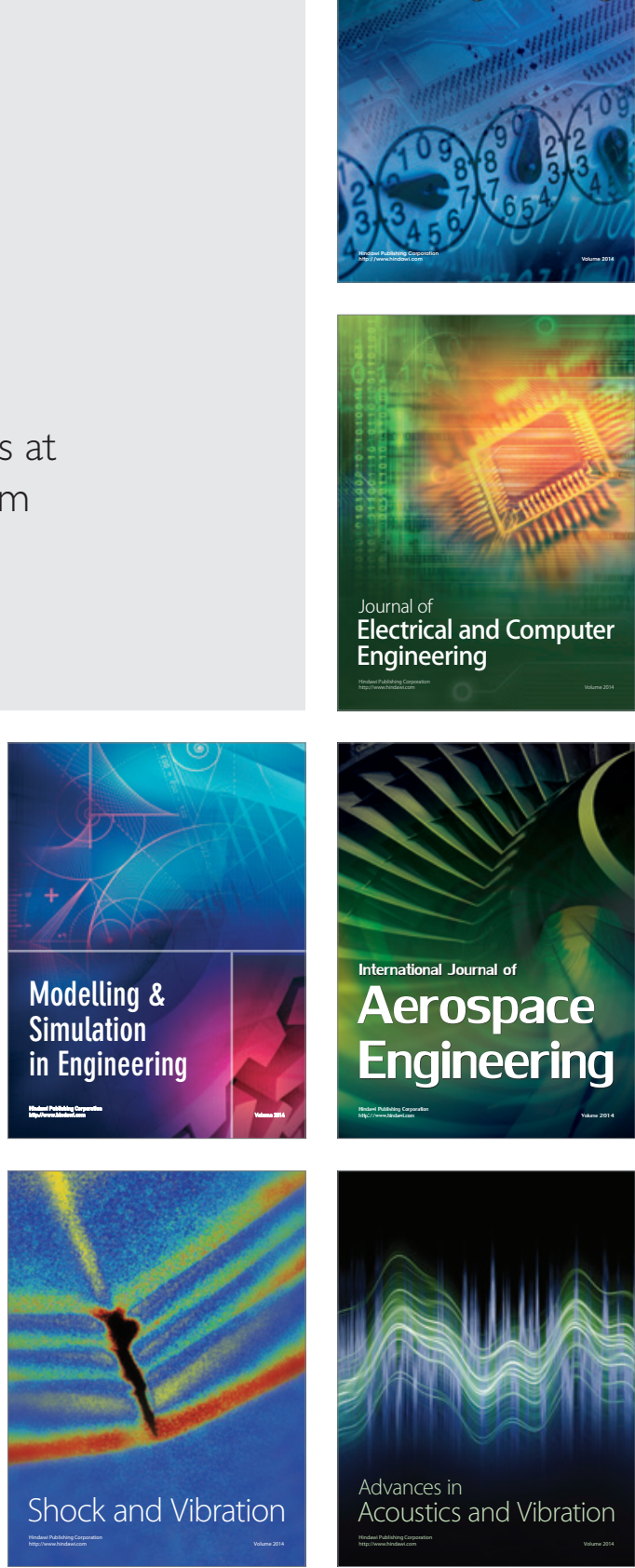